\title{
Biologia reprodutiva de Cattleya eldorado, uma espécie de Orchidaceae das campinas amazônicas*
}

\author{
Eliana Fernandez STORTI ${ }^{1}$, Pedro Ivo Soares BRAGA², Atilio STORTI FILHO ${ }^{3}$
}

RESUMO

As Orchidaceae são muito apreciadas por suas flores exóticas e exuberantes. É a maior família de plantas apresentando mais de 24000 espécies, o que denota uma alta diversidade de formas e adaptaçôes a diferentes ambientes, como também para atração, engano e manipulação de visitantes na realização da polinização cruzada. Cattleya eldorado ocorre em áreas de campinas, que são formaçóes vegetais típicas da região amazônica, que se encontram sob forte ação antrópica. Este trabalho tem como um de seus principais objetivos conhecer parte dos processos biológicos de C. eldorado fornecendo subsídios para conservá-la e manejá-la em seu habitat natural. Este estudo foi desenvolvido na Reserva Biológica de Campina, de 2000 a 2006 , durante a sua floração. C. eldorado é uma espécie epifítica que apresenta a síndrome de melitofilia, estando adaptada ao seu polinizador, a abelha Eulaema mocsaryi, que reconhece suas flores pelo odor e pelo estímulo visual através de sua coloração e reflexão de luz ultravioleta. C. eldorado é uma espécie autocompatível, embora necessite de um agente polinizador para a transferência do polinário até sua deposição na cavidade estigmática da flor.

PALAVRAS-CHAVE: Euglossina, melitofilia, orquídea.

\section{Reproductive biology of Cattleya eldorado, a species of Orchidaceae from the Amazonian white sand campinas*}

\begin{abstract}
The orchid plants are highly prized for their lush exotic flowers. It is the largest plant family with more than 24000 species, which indicates a high diversity of forms and adaptations to different environments, including the capacity to attract, deceive and manipulate visitors involved in cross-pollination. Cattleya eldorado occurs in areas of white sand campinas, a typical vegetation type of the Amazon region, which is under strong anthropogenic pressure. This work's main objectives to know the biological processes of C. eldorado providing subsidies to maintain and manage it in its natural habitat. This study was conducted from 2000 to 2006 in the Campina Biological Reserve, during its flowering period. C. eldorado is an epiphytic orchid species that has the melittophyly syndrome and is adapted to its pollinator, the bee Eulaema mocsaryi recognizing their flowers by smell and by visual stimuli, through their color and reflection of ultraviolet light. C. eldorado is self-compatible, even if it requires a pollinating agent for the transfer of the pollinarium until its deposition in the stigmatic cavity of the flower.
\end{abstract}

KEYWORDS: bees, melittophily, orchid.

\footnotetext{
* Parte do doutorado do primeiro autor.

1 Instituto Nacional de Pesquisas da Amazônia - INPA, Coordenação de Pesquisas em Ecologia, Caixa Postal 478, 69060-001, Manaus, AM. storti@inpa.gov.br

2 Universidade Federal do Amazonas - UFAM, ICB, Departamento de Biologia.

${ }^{3}$ Instituto Nacional de Pesquisas da Amazônia - INPA, Coordenação de Pesquisas em Aqüicultura.
} 


\section{INTRODUÇÃO}

A família Orchidaceae é composta por cerca de 800 gêneros e pelo menos 24000 espécies, distribuídas por todo o mundo, sendo a maior família de plantas (World Checklist of the Monocotyledons 2006). Suas flores são muito apreciadas pela diversidade de cores, formas, adaptaçôes a diferentes ambientes (Dressler 1981) e, pelas extraordinárias síndromes de polinização (Fay e Chase 2009).

O Brasil possui cerca de 2.300 espécies de orquídeas nativas, distribuídas em 190 gêneros (Pabst e Dungs 1975; 1977) e, a Amazônia brasileira apresenta catalogadas 709 espécies em 131 gêneros de orquídeas (Silva e Silva 2004).

A floresta amazônica constitui-se de um complexo vegetacional e as orquídeas ocorrem nas diversas tipologias florestais presentes. Hoehne (1949) menciona sua raridade na Floresta de Terra Firme, enquanto que Ducke e Black (1954) citam sua profusão no igapó e nas campinas. Nas várzeas amazônicas ocorrem muitas epífitas, principalmente em Hevea brasiliensis Muell. Arg. (Seringueira), um dos forófitos deste tipo vegetacional. Para os campos de terra-firme ainda existem informaçóes escassas, entretanto as florestas de galeria e ou ciliares são vias importantes de migração das orquídeas.

Dentre as diversas tipologias, as campinas se destacam por ser uma formação vegetal típica da região amazônica, principalmente da Amazônia Central e Ocidental. Elas ocupam aproximadamente $64.000 \mathrm{~km}^{2}$ (Braga 1979), cerca de 1,7\% da vegetação da Amazônia brasileira, ocorrendo em solos arenosos, pobres em nutrientes, muito ácidos, com drenagem eficiente, o lençol freático perto da superfície, o que restringe a penetração das raízes e faz com que a flora apresente altos índices de endemismo.

As Orchidaceae das campinas da Amazônia brasileira estão representadas por 155 espécies distribuídas em 64 gêneros e um híbrido natural, entre a Cattleya eldorado e a Brassavola martiana, a X Brassocattleya rubyi descoberta por P.I.S. Braga em 1977 na Reserva Biológica de Campina do INPA (Braga 1977; 1978).

Pesquisas de biologia floral em Orchidaceae no Amazonas iniciaram com o trabalho de Braga (1977) que estudou 31 espécies em uma campina da Amazônia Central. Ele verificou que a maioria das síndromes de polinizaçáo, encontrada na Reserva Biológica de Campina do INPA, mostrou adaptaçáo para insetos das ordens: Hymenoptera $(64,52 \%)$; Lepidoptera $(22,58 \%)$; Diptera $(12,9 \%)$ e beija-flores, Trochillidae $(3,22 \%)$. O primeiro atrativo para os visitantes é o aroma das flores, enquanto que a coloraçáo é uma forma secundária de atração.

Cattleya eldorado é uma espécie monofoliada pertencente à subfamília Epidendroideae, tribo Epidendreae e subtribo Laeliinae (Dressler 1981). Braga (2002) descreve dez variedades, das quais cinco estão presentes na Reserva Biológica de Campina do INPA.

Ela ocorre em uma área relativamente pequena da Amazônia, na Venezuela e no Brasil nos Estados do Amazonas e Pará, sendo que no estado do Amazonas, ela está restrita à parte central, no entorno de Manaus, nos dois lados do rio Negro, até à divisa do estado do Amazonas com Roraima. Está presente principalmente nas campinas, em áreas de alta luminosidade, vegetando preferencialmente sobre o macucu (Aldina heterophylla Spr. ex Benth., Fabaceae) que possui uma casca bem rugosa, nos igapós e, raramente, na terra-firme (Lacerda 1995).

Os principais forófitos da Cattleya eldorado na Reserva Biológica de Campina são: Aldina heterophylla Spruce ex Benth. (Leguminosae), Pagamea duckei Standley (Rubiaceae), Pradosia schomburgkiana A.DC. Cronq. (Sapotaceae), Manilkara amazonica (Huber) Standl. (Sapotaceae) e Eugenia sp. (Myrtaceae) (Storti 2007).

Segundo Braga (1977), o polinizador da C. eldorado é a abelha Eulaema (Apeulaema) mocsaryi Friese (Euglossina). Esta abelha foi observada por ele em apenas duas ocasiōes, durante todo o período de estudo, tendo sido atraída primariamente pelo odor e coloraçáo das flores.

Neste sentido, este trabalho tem como principais objetivos conhecer parte dos processos biológicos de Cattleya eldorado Linden, tais como a biologia floral, polinizaçáo e o sistema reprodutivo e; fornecer subsídios para conservá-la e manejá-la em seu habitat natural, as campinas da Amazônia, áreas que se encontram sob forte ação antrópica.

\section{MATERIAL E MÉTODOS}

\section{Área de estudo}

As observaçôes de campo foram realizadas de março de 2000 a janeiro de 2006 na Reserva Biológica de Campina, pertencente à Estação Experimental de Silvicultura Tropical do INPA, AM ( $\left.2^{\circ} 35^{\prime} 25,9^{\prime \prime} \mathrm{S}-60^{\circ} 01^{\prime} 49,5^{\prime \prime} \mathrm{W}\right)$, situada no $\mathrm{km} 45$ da rodovia BR 174, que liga Manaus a Boa Vista. A Reserva Biológica de Campina do INPA mede aproximadamente 900 hectares, mas apenas dois deles são de vegetaçấo de campina. As características de uma vegetação de campina sáo: a presença de árvores baixas de ramificação tortuosa, casca rugosa, esclerofilia e a presença de epífitas em abundância. Este trabalho foi realizado em uma área de campina sombreada, segundo a terminologia apresentada por Anderson et al. (1975), onde ocorre a C. eldorado. O clima, segundo a classificação de Köppen, é do tipo Afi: A - Clima tropical praticamente sem inverno, com temperatura média para o mês mais frio nunca inferior a $18^{\circ} \mathrm{C} ; f$ - chuvas durante todo o ano; i - indica isotermia, ou seja, as oscilaçôes anuais de temperatura média não chegam a $5^{\circ} \mathrm{C}$; não há verão nem 
inverno (Ribeiro 1976). A formaçáo vegetacional da área é arbustivo-arbórea-graminóide, o que difere bastante das imponentes florestas amazônicas, localizada sobre um solo podzólico em manchas de areia branca (Ferreira 1997).

\section{Biologia floral e polinização}

Foram feitas análises da morfologia floral com auxílio de um esteromicroscópio binocular, com equipamento fotográfico acoplado, em 10 flores frescas de cada uma das variedades de Cattleya eldorado coletadas na área de estudo, sendo medidos o comprimento da sépala, pétala, labelo e coluna, como também o diâmetro do labelo. Foi verificado o número de flores em 272 inflorescências de C. eldorado, como também a porcentagem de cada variedade em 100 inflorescências ensacadas ao acaso, na fase de botáo. A longevidade floral foi observada em 113 flores (100 indivíduos).

A receptividade do estigma foi verificada em 3 flores intactas de cada variedade de $C$. eldorado, com o auxílio de uma lupa manual. A técnica de Macior (1986), utilizando o peróxido de hidrogênio a 3\%, foi aplicada para uma melhor visualização, através da atividade da peroxidase, pelo borbulhamento sobre o estigma.

Dados sobre a reflexão de luz ultravioleta nas flores foram obtidos utilizando-se hidróxido de amônia $\left(\mathrm{NH}_{4} \mathrm{OH}\right)$ segundo a técnica de Gertz (1938). Três flores de cada variedade foram colocadas em frasco contendo o hidróxido de amônia por 10 minutos. A emissão de odor foi detectada utilizando-se o vermelho neutro $\left(\mathrm{C}_{15} \mathrm{H}_{17} \mathrm{~N}_{4} \mathrm{Cl}\right)$ segundo a técnica apresentada por Vogel (1962). As flores testadas (três de cada variedade) foram mergulhadas em solução aquosa de vermelho-neutro e água destilada (1:10000) por 30 minutos e, após este período, lavadas em água corrente.

Lâminas de pólen das cinco variedades de C. eldorado foram confeccionadas no Laboratório de Palinologia do INPA. Testes de fertilidade potencial dos grãos de pólen foram realizados em políneas de 10 flores de cada variedade. As políneas eram maceradas individualmente sobre uma lâmina com tetrazólio $(0,2 \%$ de cloreto 2,3,5-trifeniltetrazolium) segundo Lee (1967). Foram montadas 10 lâminas de cada variedade e, em cada uma, observados 20 campos ao acaso.

Foi realizado um total de 160 horas de campo, incluindo observação do comportamento dos visitantes nas flores da C. eldorado. Exemplares dos visitantes florais coletados estâo depositados na Coleção de Invertebrados do INPA. Inflorescências de 100 plantas foram marcadas na fase de botão floral e as visitas dos polinizadores também foram acompanhadas de modo indireto, através da ausência de polinário, desde a abertura da flor até sua senescência ou até o início da formação do fruto.

\section{Sistema reprodutivo}

O sistema reprodutivo de $C$. eldorado foi estudado através de autopolinização espontânea, autopolinização manual, polinização cruzada e a verificação de ocorrência ou não de agamospermia com a retirada do polinário (Radford $e t$ al. 1974), em 263 flores selecionadas aleatoriamente de 272 inflorescências de plantas, localizadas em diferentes forófitos da Reserva Biológica de Campina do INPA. Os testes foram realizados em flores no primeiro dia de antese usando sacos de filó de malha fina $(<1 \mathrm{~mm}$ de diâmetro). As flores foram previamente ensacadas no estágio de botão e depois de realizado o teste em questáo foram novamente ensacadas evitando, desta forma, o contacto com possíveis animais visitantes. O desenvolvimento de 222 flores (em 100 inflorescências) não ensacadas (controle) foi monitorado para verificar a taxa de produção natural de cápsulas em C. eldorado.

A viabilidade das sementes dos frutos formados nas experiências para se verificar o sistema reprodutivo de $C$. eldorado, foi observada utilizando-se o tetrazólio segundo a técnica de Lee (1967), de maneira semelhante à que foi usada para os grãos de pólen. Para a análise das sementes foram consideradas viáveis aquelas em que o embriáo era corado pelo tetrazólio.

\section{RESULTADOS E DISCUSSÃO \\ Biologia floral e polinização}

A floração desta espécie de orquídea começa a partir do mês de outubro indo até março do ano seguinte, sendo que o pico de floração ocorre nos meses de novembro e dezembro.

Cattleya eldorado é uma espécie de orquídea epifítica, unifoliada, com folhas coriáceo-carnosas, oblongas e verdes. Sua inflorescência é do tipo simples, racemosa, ereta e pauciflora. As 272 inflorescências estudadas apresentaram de uma a sete flores, com uma média de 2,04 $\pm 1,04$ flores por inflorescência, que estão inseridas no ápice do pseudobulbo (Figura 1).

$\mathrm{Na}$ Reserva estáo presentes cinco, das dez variedades de C. eldorado, descritas por Braga (2002): C. eldorado var. alba Rand.; C. eldorado var. eldorado Linden; C. eldorado var. oweni Hort.; C. eldorado var. splendens Linden e C. eldorado var. treyeranae Linden. A porcentagem de ocorrência dessas variedades encontra-se na Tabela 1.

As flores de C. eldorado apresentam duas sépalas laterais falcadas e uma sépala dorsal, oblonga acuminada. As duas pétalas são ovadas e apiculadas no ápice; o labelo é membranáceo, séssil e trilobado. A coluna é branca, raras vezes de tons violáceos; o polinário apresenta dois pares de 


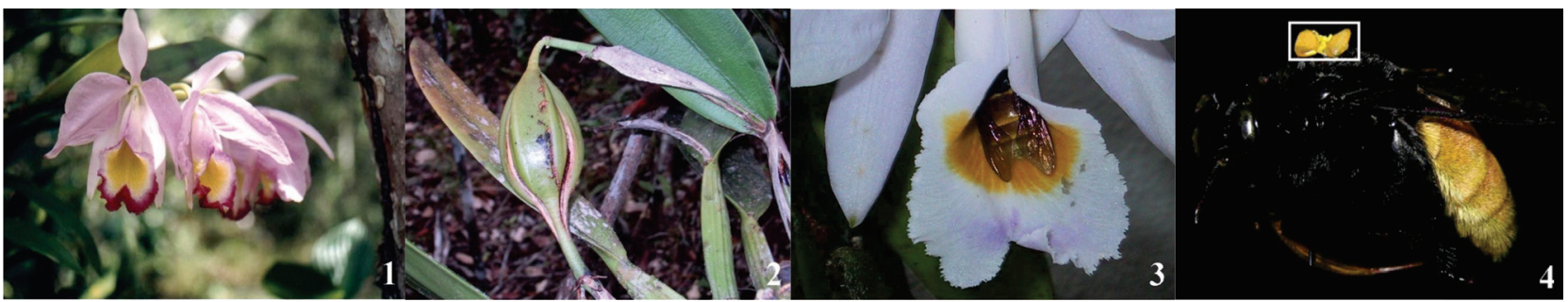

Figuras 1 a 4 - Cattleya eldorado. 1. Inflorescência. 2. Cápsula. 3. Eulaema mocsaryi visitando a flor. 4. Polinário aderido à abelha E. mocsaryi.

políneas amarelas e ceróides e também possui nectário tubular ao lado do ovário.

Das 100 inflorescências ensacadas ao acaso na fase de botão, verificamos que duas não chegaram a se desenvolver por danos causados por formigas nos botóes florais. $\mathrm{O}$ tamanho das peças florais das quatro variedades e da espécie tipo são semelhantes, apenas a variedade alba apresentou flores um pouco menores (Tabela1).

As flores duram em média de seis a 10 dias e, ocorrendo a fecundação já podemos observar o início da formação do fruto, pela dilatação do ovário.

O fruto de Cattleya eldorado é do tipo cápsula e contém milhares de sementes muito pequenas e amarelas em seu interior (Figura 2). A deiscência dos frutos começa de nove a 10 meses após a fecundação. Ao abrir, a flor de C. eldorado apresenta uma substância viscosa na cavidade estigmática. Esta substância é depositada, com o auxílio do rostelo, sobre o polinizador permitindo a fixação das políneas em seu corpo.

Com o uso do peróxido de hidrogênio a 3\% foi observado borbulhamento na cavidade estigmática, tanto em flores recém abertas como em flores com mais de 16 dias de duração, indicando a atividade da peroxidase durante todo este período. A presença desta enzima indica a receptividade do estigma (Kearns e Inoye 1993).

As flores testadas com hidróxido de amônia, para se verificar o seu padrão de reflexão à luz ultravioleta, apresentaram uma coloração intensa de cor amarelo nas sépalas, pétalas e labelo. Não houve diferenças significativas entre as cinco variedades. Estas partes que se coram de amarelo são regióes que refletem a luz ultravioleta. Padróes de ultravioleta têm um papel de grande significado ecológico na visão das abelhas funcionando, à curta distância, como guias de recompensa para elas (Barth 1991).

As flores de Cattleya eldorado exalam um odor agradável e suave, sua emissão é contínua durante a floração sendo mais intenso principalmente pela manhã. Com a utilização do vermelho-neutro observou-se que as pétalas e o labelo coraram mais intensamente que as sépalas, que coraram mais fortemente no ápice, indicando assim a localização das células produtoras de odor. $\mathrm{O}$ padrão de coloraçáo das flores nas cinco variedades foi semelhante.

Em Cattleya eldorado os grãos de pólen são produzidos em tétrades, sendo que suas paredes estão dispostas de várias maneiras, resultado dos vários arranjos possíveis das células nas tétrades. Apenas $40 \%$ dos grãos foram corados nas lâminas com o tetrazólio.

Algumas plantas de C. eldorado apresentaram danos nas folhas, pseudobulbos, botóes florais e nas flores, causados por insetos e fungos.

Duas espécies de formigas do gênero Trachymyrmex sp.1 e sp. 2 (Myrmicinae, Attini) foram observadas recortando sépalas, pétalas e o labelo danificando muitas flores de $C$. eldorado, como também botóes florais. Estas espécies de formigas são cortadeiras e alimentam-se de fungos que cultivam em seus ninhos (José M. S. Vilhena com. pess.).

Também foi observada a presença de larvas de Diptera se alimentando dos tecidos da coluna da flor, comprometendo toda a cavidade estigmática.

Tabela 1- Porcentagem de ocorrência e tamanho das peças florais das variedades de Cattleya eldorado Linden, na Reserva Biológica de Campina do INPA. Média $(\mathrm{cm}) \pm$ Desvio Padrão.

\begin{tabular}{lcccccc}
\hline $\begin{array}{l}\text { Variedades de } \\
\text { Cattleya eldorado }\end{array}$ & $\begin{array}{c}\text { Ocorrência } \\
(\%)\end{array}$ & Sépala & Pétala & Labelo & $\begin{array}{c}\text { Labelo } \\
\text { (Diâmetro) }\end{array}$ & Coluna \\
\hline alba & 4 & $6,52 \pm 0,33$ & $6,41 \pm 0,52$ & $6,12 \pm 0,56$ & $1,33 \pm 0,11$ & $3,01 \pm 0,12$ \\
eldorado & 34 & $6,56 \pm 0,59$ & $6,49 \pm 0,44$ & $6,10 \pm 0,61$ & $1,48 \pm 0,19$ & $3,06 \pm 0,27$ \\
oweni & 6 & $6,58 \pm 0,31$ & $6,55 \pm 0,17$ & $6,17 \pm 0,24$ & $1,50 \pm 0$ & $3,07 \pm 0,34$ \\
splendens & 20 & $6,45 \pm 0,41$ & $6,59 \pm 0,54$ & $6,29 \pm 0,62$ & $1,47 \pm 0,06$ & $2,93 \pm 0,27$ \\
treyeranae & 34 & $5,92 \pm 0,64$ & $6,15 \pm 0,58$ & $5,66 \pm 0,49$ & $1,47 \pm 0,17$ & $2,96 \pm 0,45$ \\
\hline
\end{tabular}


A presença de fungos, em algumas plantas de C. eldorado, causou manchas nas folhas e nos pseudobulbos levando à contaminação de toda planta.

Outros insetos, como por exemplo, gafanhotos, baratas, besouros, formigas e abelhas e até mesmo aranhas, foram ocasionalmente observados nas flores da Cattleya eldorado. Os gafanhotos e as baratas (Orthoptera) estavam se alimentando de peças florais. Os coleópteros das famílias Curculionidae e Chrysomelidae também foram observados nas flores. Em algumas espécies de Laelia e Cattleya, as fêmeas de besouros Diorymerellus lepagei e $D$. minensis costumam perfurar o ovário para colocarem seus ovos. Suas larvas se alimentam das partes internas da flor levando-a à destruição (Vendramin et al. 2002).

A formiga Pachycondyla villosa inversa (Ponerinae, Ponerini), que é uma espécie carnívora, foi observada caminhando sobre as flores, provavelmente à captura de seu alimento.

Um indivíduo de Exaerete sp. (Apidae, Euglossina) foi observado penetrando rapidamente em uma flor de C. eldorado e, logo após se dirigiu a uma flor de outra espécie de orquídea, a Prostechea fragrans. Nesta rápida visita, o polinário não foi retirado da antera. As abelhas do gênero Exaerete são grandes e de colorido verde metálico; todas são parasitas de ninhos de Eulaema e Eufriesea (Silveira et al. 2002).

A aranha caranguejo Thomisus sp. (Aranae, Thomiidae) foi observada capturando uma abelha Plebeia minima no interior do labelo da $C$. eldorado.

A abelha Plebeia minima (Apidae, Meliponina) foi observada penetrando na base da antera, provavelmente para coletar grãos de pólen do interior das políneas de C. eldorado.

Um indivíduo de Euglossa sp. (Apidae, Euglossina) foi observado visitando uma flor de $C$. eldorado por aproximadamente 20 minutos. Ele pousa no labelo e se locomove por toda sua extensão em movimentos repetidos de entrada e saída da flor. Em nenhum destes movimentos o polinário foi retirado da antera. Esta é uma abelha pequena e seu corpo não possui dimensóes para o contacto com o polinário.

$\mathrm{Na}$ Reserva Biológica de Campina do INPA, a abelha Eulaema mocsaryi (Friese 1899) (Apidae, Euglossina) foi observada em quatro ocasióes sobrevoando a área onde se encontravam as flores da Cattleya eldorado. A presença desta abelha ocorreu entre 10:00 e 12:30h aproximadamente (Figuras 3 e 4 ).

Em apenas duas das ocasióes foi observado o mecanismo de polinizaçáo desta espécie de abelha. Ao se aproximar da flor de C. eldorado, atraída pelo odor e cores desta espécie de orquídea, E. mocsaryi pousa no labelo locomovendo-se em direção ao seu interior, forçando sua entrada. Ela coleta substâncias odoríferas provavelmente raspando o interior do labelo com os tarsos de suas patas anteriores e, ao sair da flor, esbarra seu corpo no rostelo permitindo a deposição de uma substância pegajosa presente na cavidade estigmática, na parte superior do tórax, onde as políneas irão se aderir.

Ao sair da flor ela transfere as substâncias odoríferas coletadas com as patas anteriores, para as medianas e posteriormente para as tíbias das patas posteriores. Neste local existem fendas cobertas de pelos em que as substâncias odoríferas serão absorvidas. O tempo de permanência na flor foi de aproximadamente 20 segundos.

Braga (1977) também observou esta espécie de abelha em flores de C. eldorado em duas ocasióes, sendo que o seu tempo de permanência na flor foi de 50 segundos.

O reconhecimento da flor da $C$. eldorado pela abelha Eulaema mocsaryi se faz pelo odor, consistindo no seu atrativo primário. Ao aproximar-se dela o reconhecimento se faz pelo estímulo visual, cor, forma e reflexão de ultravioleta, que são os atrativos secundários. Acredita-se que a coleta de odores pelos machos de Euglossina esteja relacionada com as atividades reprodutivas destas abelhas, provavelmente como precursor de seu próprio feromônio sexual (Dressler 1981; Williams e Whitten 1983).

Durante o período de estudo não houve polinizaçóes noturnas uma vez que não foi observada a presença de políneas aderidas à cavidade estigmática, como também não houve a retirada de polinário nas flores de Cattleya eldorado marcadas após as 16 horas.

Do acompanhamento de 100 plantas para a observação da taxa de visitas as flores de C. eldorado, em condiçóes naturais, foram formadas 224 flores e constatou-se que $25,9 \%$ das flores foram visitadas, ocorrendo um sucesso de 5,3\% de frutos formados em relaçáo ao total de flores e um sucesso de $20,7 \%$ em relação ao total de flores visitadas (Tabela 2). Foram observados políneas aderidas em cavidades estigmáticas de três flores de C. eldorado.

Durante o período de observação, muitas flores (22,3\%) foram danificadas pelo ataque de formigas e coleópteros ou

Tabela 2 - Taxa de visita nas variedades de flores da Cattleya eldorado Linden, na Reserva Biológica de Campina do INPA.

\begin{tabular}{lccc}
\hline \multirow{2}{*}{$\begin{array}{l}\text { Variedade de Cattleya } \\
\text { eldorado }\end{array}$} & \multicolumn{2}{c}{ Flor } & \multirow{2}{*}{ Fruto } \\
\cline { 2 - 3 } & Visitada & Não visitada & \\
\hline alba & 8 & 2 & 2 \\
eldorado & 28 & 58 & 4 \\
oweni & 4 & 8 & 2 \\
splendens & 6 & 30 & 0 \\
treyeranae & 12 & 68 & 4 \\
\hline TOTAL & 58 & 166 & 12 \\
\hline
\end{tabular}


apresentaram alguma deformação na coluna tornando-as menos atrativas aos polinizadores

\section{Sistema reprodutivo}

Os tratamentos para caracterização do sistema reprodutivo da Cattleya eldorado mostraram que ela é uma espécie autocompatível, mesmo não tendo sido formados frutos por autopolinizaçóes espontâneas (Tabela 3).

Muitas espécies de orquídeas são autocompatíveis, mas devido a barreiras espaciais, ecológicas e mecânicas a polinização cruzada é favorecida (Pijl e Dodson 1969).

A presença do rostelo em C. eldorado, como em outras espécies de orquídeas, funciona como uma barreira mecânica, dificultando ou até mesmo impossibilitando o contacto entre o polinário e a cavidade estigmática evitando desta forma a autopolinização.

Através da autopolinização manual verificou-se que 41,5\% das flores testadas produziram frutos indicando que, embora C. eldorado seja uma espécie autocompatível, ela necessita de um agente polinizador para a transferência do polinário até sua deposição na cavidade estigmática.

A autogamia é relativamente rara em orquídeas, aproximadamente 3\% das espécies são autógamas (Pijl e Dodson 1969). Dentro do gênero Cattleya ocorre uma espécie que se autofecunda, a $C$. aurantiaca, e uma que mostra tendência à autopolinização, a $C$. deckerii (Standley 1942). Na C. aurantiaca a autogamia pode ocorrer em botóes fechados, botóes parcial ou totalmente abertos, onde nas flores autopolinizadas ocorre a autólise do rostelo precedendo a polinizaçáo permitindo a queda da polínea no estigma (Arditti 1992).

Apenas um fruto apomítico começou a se formar, mas não chegou até o final de seu desenvolvimento. Apomixia ocorre em algumas orquídeas de zonas temperadas, mas está contra a tendência em direção à polinização cruzada pela família (Pijl e Dodson 1969).

A pequena quantidade de frutos formados em condiçóes naturais $(3,1 \%)$, como também o observado por Storti (2007) em três amostras (Anos 2000 - 2001, Anos 2002 - 2003 e Anos 2004 - 2005) 7,9; 3,6 e 3,9\%, respectivamente, provavelmente está relacionada, como em muitas espécies de

Tabela 3 - Resultado dos tratamentos para avaliar o sistema reprodutivo da Cattleya eldorado Linden, na Reserva Biológica de Campina do INPA.

\begin{tabular}{lccc}
\hline Teste & $\begin{array}{c}\text { Flores } \\
(\mathrm{n})\end{array}$ & $\begin{array}{c}\text { Frutos } \\
(\%)\end{array}$ & $\begin{array}{c}\text { Viabilidade das } \\
\text { Sementes (\%) }\end{array}$ \\
\hline Agamospermia & 50 & 0 & 0 \\
Autopolinização espontânea & 50 & 0 & 0 \\
Autopolinização manual & 53 & 41,5 & 28,8 \\
Polinização cruzada & 53 & 75,5 & 53,5 \\
Polinização natural (controle) & 222 & 3,1 & 54,3 \\
\hline
\end{tabular}

orquídeas não autógamas, à transferência de pólen deficiente devido à escassez de polinizadores (Ackerman 1989).

Muitos frutos que começaram a ser formados não chegaram ao final de seu desenvolvimento. Houve uma taxa de aproximadamente $56 \%$ de perda de frutos por autopolinização manual, $17 \%$ por xenogamia e $59 \%$ em condiçóes naturais.

$\mathrm{O}$ aborto de frutos pode ocorrer por diversas razóes como, por exemplo, pólen náo viável, mecanismos de incompatibilidade, ataque de patógenos, herbivoria, predação, entre outros (Ackerman 1989).

Em C. eldorado foram observados frutos com perfuraçóes, provavelmente por coleópteros Curculionidae, estes besouros podem estar parasitando o ovário, onde as suas larvas se desenvolvem. Também foram observados frutos enegrecidos pela presença de fungos. Outros estudos deverão ser realizados a fim de se identificar a causa de abortos em frutos de $C$. eldorado.

A porcentagem da viabilidade das sementes dos 86 frutos formados nos tratamentos de autopolinização manual e polinizaçóes cruzadas encontra-se na Tabela 3. A viabilidade das sementes provenientes de polinização cruzada (xenogamia) e a dos frutos produzidos naturalmente foram muito próximas, $53,5 \%$ e $54,3 \%$, respectivamente, enquanto que a de autopolinização manual foi de $28,8 \%$.

$\mathrm{Na}$ natureza, as sementes de Cattleya são geralmente infectadas por um fungo que lhes fornece nutrientes necessários para sua germinação e início do crescimento (Chadwick e Chadwick 2006).

Stort e Martins (1980) estudando a autopolinização e a polinização cruzada, em 15 espécies de Cattleya verificaram que, de um modo geral, a frequência de sementes com embriáo não é alta, mesmo em flores em que foi realizada a fecundação cruzada, mas a autofecundaçáo pode ser realizada com êxito e utilizada no melhoramento destas orquídeas.

\section{CONCLUSÕES}

Cattleya eldorado é uma espécie melitófila e a abelha Eulaema mocsaryi foi considerada sua polinizadora na Reserva Biológica de Campina do INPA, por seu comportamento, ao ser atraída pelo odor e coloraçáo de suas flores.

O sistema reprodutivo empregado por esta espécie de orquídea é a xenogamia e; a baixa produção natural de frutos ocorre pela escassez do polinizador e pelos danos causados por insetos e fungos nas suas flores e frutos.

Uma vez conhecidas a biologia floral, a polinização, o sistema reprodutivo, como também a dinâmica da população da C. eldorado na Reserva Biológica de Campina do INPA, já se tem informaçóes suficientes de sua reintrodução na própria reserva, e também de como conservá-la em seu habitat natural, as campinas amazônicas. 


\section{AGRADECIMENTOS}

Ao Dr. Roger W. Hutchings pela colaboração constante e, à Coordenação de Pesquisas em Ecologia (CPEC) do Instituto Nacional de Pesquisas da Amazônia pelo apoio logístico.

\section{BIBLIOGRAFIA CITADA}

Ackerman, J.D. 1989. Limitations to sexual reproduction in Encyclia krugii (Orchidaceae). Systematic Botany, 14: 101-109.

Anderson, A.; Prance, G.T.; Albuquerque, B.W.P. 1975. Study on the vegetation of the Amazonian Campinas III - The woody vegetation from Campina Biological Reserve INPA - SUFRAMA (Manaus - Caracaraí, km 62). Acta Amazonica, 5: 225-246.

Arditti, J. 1992. Fundamentals of orchid biology. New York, John Wiley \& Sons. 691 pp.

Barth, F.G. 1991. Insects and flowers. The biology of a partnership. New Jersey, Princeton University Press. 408 pp.

Braga, P.I.S. 1977. Biological aspects of the Orchidaceae from a Central Amazonian Campina. Acta Amazonica, 7: supl. 89 pp.

Braga, P.I.S. 1978. Studies of flora orchidology Amazonas State III $\mathrm{X}$ Brassocattleya rubyi Braga (Orchidaceae) a new natural hybrid of the Amazon flora. Acta Amazonica, 8: 371-378.

Braga, P.I.S. 1979. Phytogeographical subdivision, vegetation types, floristic and conservation of the Amazon rainforest. Acta Amazonica, 9: 53-80. supl.

Braga, P.I.S. 2002. Cattleya eldorado Linden and its varieties. (www. delfinadearaujo.com). Acesso em 22/04/2002.

Chadwick, A.A.; Chadwick, A.E. 2006. The classic Cattleyas. Portland, Timber Press. 251 pp.

Dressler, R.L. 1981. The orchids, natural history and classification. Cambridge, Harvard University Press. 332 pp.

Ducke, A.; Black, G.A. 1954. Notes on the phytogeography of Brazilian Amazon. Boletim Técnico do Instituto Agronômico, 29: $1-62$.

Fay, M.F.; Chase, M.W. 2009. Orchid biology: from Linnaeus via Darwin to the $21^{\text {st }}$ century. Annals of Botany, 104: 359-364.

Ferreira, C.A.C. 1997. Physionomic floristic variation and vegetation transition, campina, campinarana and upland forest in central Amazonia, Manaus (AM). Dissertação de Mestrado. Curso de Pós-Graduação da Universidade Federal Rural de Pernambuco, Recife, Pernambuco. 112 pp.

Gertz, O. 1938. Ueber die Verbreitung des Anthochlors bei den Compositen. Kgl. Fysiogr. Sällsk. Lund. Förh., 8: 62-70. (Apud Scogin, R.; Young, D.A.; Jones Jr., C.E. 1977. Anthochlor pigments and pollination biology. II. The ultraviolet floral pattern of Coreopsis gigantea (Asteraceae). Bulletin of the Torrey Botanical Club, 104: 155-159).

Hoehne, F.C. 1949. Orchidaceae iconography of Brazil. São Paulo, Secretaria da Agricultura. 301 pp.

Kearns, C.A.; Inouye, D.W. 1993. Techniques for pollination biologists. University Press of Colorado, Colorado. 583 pp.
Lacerda, K.G. 1995. Amazon discovery of new species and extinction, p.8-123. In: Lacerda, K.G. et al. (eds). Brazilian Orchids. Sodo Publishing.

Lee, A.E. 1967. Growth and development of plants. São Paulo, EDART Livraria Editora Ltda. 96 pp.

Macior, LW. 1986. Floral resource sharing by bumblebees and hummingbirds in Pedicularis (Scrophulariaceae) pollination. Bulletin of the Torrey Botanical Club, 113: $101-109$.

Pabst, G.F.J.; Dungs, F. 1975. Orchidaceae brasilienses, I. Hildesheim, Brücke-Verlag Kurt Schmersov. 408 pp.

Pabst, G.F.J.; Dungs, F. 1977. Orchidaceae brasilienses, II. Hildesheim, Brücke-Verlag Kurt Schmersov. 418 pp.

Pijl, L. van der; Dodson, C.H. 1969. Orchid flowers. Their pollination and evolution. University of Miami Press, Florida. 214 pp.

Radford, A.E.; Dickison, W.C.; Massey, J.R.; Ritchie Bell, C. 1974. Vascular plant systematics. New York, Harper \& Row Publishing. $891 \mathrm{pp}$.

Ribeiro, M.N.G. 1976. Climatological aspects of Manaus. Acta Amazonica, 6: 229-233.

Silva, M.F.F.; Silva, J.B.F. 2004. Native Orchids of the Brazilian Amazon II. Belém, Universidade Federal Rural do Pará, Museu Paraense Emílio Goeldi. 540 pp.

Silveira, F.A.; Melo, G.A.R.; Almeida, E.A.B. 2002. Brazilian bees: systematics and identification. Belo Horizonte, Ministério do Meio Ambiente. 253 pp.

Standley, P. 1942. Flora of the Panama. Cand. Contr. U. S. Nath. Herb., 27: 141. (Apud Stort \& Martins, 1980 op cit.).

Stort, M.N.S.; Martins, P.S. 1980. Self-pollination e cross pollination in some species of the genus Cattleya (Orchidaceae). Ciência e Cultura, 32: 1080-1083.

Storti, E.F. 2007. Population dynamics and the reproductive biology of Cattleya eldorado Linden (Orchidaceae). Tese de Doutorado, Intituto Nacional de Pesquisas da Amazônia/Universidade Federal do Amazonas, Manaus, Amazonas. 131 pp.

Vendramin, J.D.; Parra, J.R.P.; Souza, A.P. 2002. Insects and other pests of orchids and their control, p. 43-57. In: Gioria, R. (org.). $D$ \& $P$ that attack orchids. São Paulo, Brasil.

Vogel, S. 1962. Duftdrüsen im Dienste der Beestäubung. Akad. Wiss. Abh. Math. Naturwiss. Kl., 10: 599-763. (Apud Webber, A.C. 1981. Floral biology of some Annonaceae in the Manaus region, AM. Dissertação de Mestrado, Intituto Nacional de Pesquisas da Amazônia/Universidade Federal do Amazonas, Manaus. 83 pp).

Williams, N.H.; Whitten, W.M. 1983. Orchid floral fragrances and male euglossine bees: Methods and advances in the last sesquidecade. The Biological Bulletin, 164: 355-395.

World Checklist of Monocotyledons. 2006. The board of trustees of the Royal Botanic Gardens, Kew. (www.kew.org). Acesso em 02/09/2009.

Recebido em 08/04/2010

Aceito em 30/09/2010 